Received: 23 August 2017

Accepted: 9 October 2017

Published online: 27 October 2017

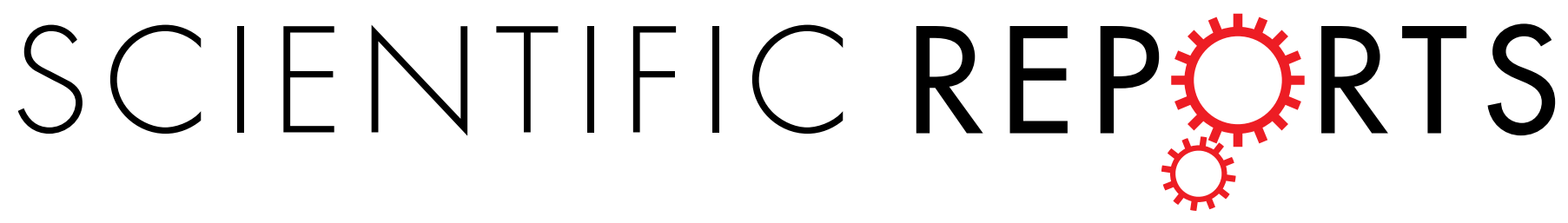

\title{
OPEN A Hybrid Gate Dielectrics of Ion Gel with Ultra-Thin Passivation Layer for High-Performance Transistors Based on Two-Dimensional Semiconductor Channels
}

\author{
Hyunjin Jo ${ }^{1}$, Jeong-Hun Choi ${ }^{1}$, Cheol-Min Hyun ${ }^{1}$, Seung-Young Seo ${ }^{2}$, Da Young Kim ${ }^{3}$, \\ Chang-Min Kim ${ }^{3}$, Myoung-Jae Lee $\mathbb{1}^{4}{ }^{4}$, Jung-Dae Kwon ${ }^{5}$, Hyoung-Seok Moon 6 , Se-Hun Kwon ${ }^{3}$ \& \\ Ji-Hoon Ahn ${ }^{1}$
}

We propose a hybrid gate structure for ion gel dielectrics using an ultra-thin $\mathrm{Al}_{2} \mathrm{O}_{3}$ passivation layer for realizing high-performance devices based on electric-double-layer capacitors. Electric-double-layer transistors can be applied to practical devices with flexibility and transparency as well as research on the fundamental physical properties of channel materials; however, they suffer from inherent unwanted leakage currents between electrodes, especially for channel materials with low off-currents. Therefore, the $\mathrm{Al}_{2} \mathrm{O}_{3}$ passivation layer was introduced between the metal electrodes and ion gel film as a leakage current barrier; this simple approach effectively reduced the leakage current without capacitance degradation. In addition, we confirmed that a monolayer $\mathrm{MoS}_{2}$ transistor fabricated with the proposed hybrid gate dielectric exhibited remarkably enhanced device properties compared to a transistor using a normal ion gel gate dielectric. Our findings on a simple method to improve the leakage current properties of ion gels could be applied extensively to realize high-performance electric-double-layer transistors utilizing various channel materials.

Ionic liquids provide the advantage of very large capacitances by the formation of nanogap capacitors at solidliquid interfaces ${ }^{1,2}$, and electric double-layer-transistors (EDLTs) using ionic liquids have received significant attention because they show ultra-high capacitance and potential applicability in both practical flexible and transparent devices and studies on the fundamental physical properties of channel materials, including carrier transport phenomena, superconductivity, and thermoelectricity ${ }^{3-13}$. Despite this broad applicability, ionic liquids are limited in application to practical devices because of fluidity-related instability; however, this problem can be solved by introducing an ion gel, comprising a mixture of an ionic liquid and a polymer network, which maintains both the mechanical flexibility of the polymer and the large specific capacitance of the ionic liquid ${ }^{14-19}$. As demonstrated by printable and photo-patternable ion gels, large-area device integration and the realization of practical applications of stretchable and flexible devices have become possible $e^{20,21}$.

\footnotetext{
${ }^{1}$ Department of Electronic Material Engineering, Korea Maritime and Ocean University, 727 Taejong-ro, Yeongdo-gu, Busan, 49112, Republic of Korea. 'Department of Material Science and Engineering, Pohang University of Science and Technology, 77 Cheongam-Ro, Pohang, 790-784, Republic of Korea. ${ }^{3}$ School of Materials Science and Engineering, Pusan National University, 30 Jangjeon-Dong Geumjeong-Gu, Busan, 609-735, Republic of Korea. ${ }^{4}$ Daegu Gyeongbuk Institute of Science and Technology (DGIST), 333 Techno Jungang Daero, HyeonpungMyeon, Dalseong-Gun, Daegu, 42988, Republic of Korea. ${ }^{5}$ Department of Advanced Functional Thin Films, Surface Technology Division, Korea Institute of Materials Science, 797 Changwondaero, Sungsan-Gu, Changwon, Gyeongnam, 51508, Republic of Korea. ${ }^{6}$ Energy Plant R\&D Group, Korea Institute of Industrial Technology (KITECH), 30 Gwahaksandan 1-ro 60beon-gil, Gangseo-gu, Busan, 46742, Republic of Korea. Hyunjin Jo, Jeong-Hun Choi and Cheol-Min Hyun contributed equally to this work. Correspondence and requests for materials should be addressed to S.-H.K. (email: sehun@pusan.ac.kr) or J.-H.A. (email: ajh1820@kmou.ac.kr)
} 
(a)

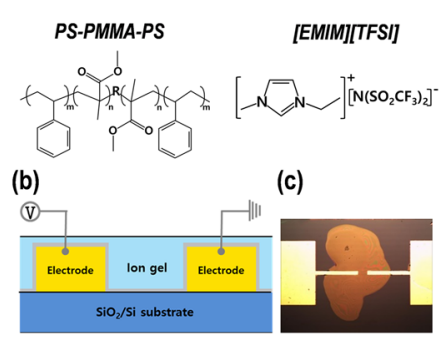

(d)

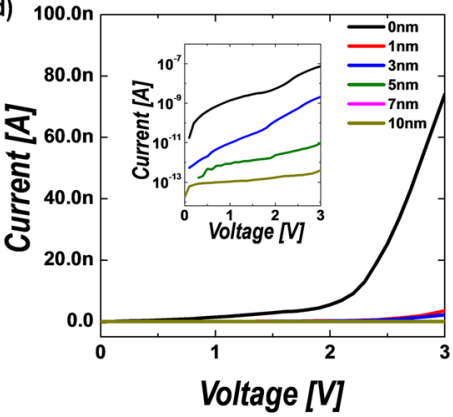

(e)

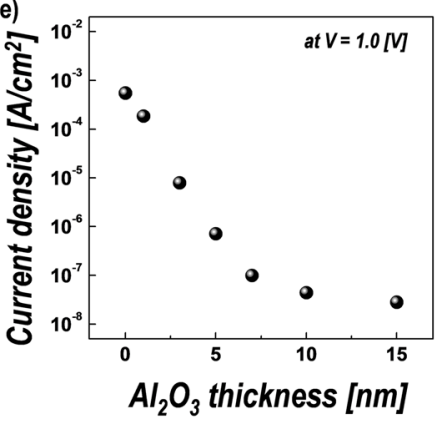

Figure 1. (a) Molecular structures of the triblock copolymer (PS-PMMA-PS) and ionic liquid ([EMIM] [TFSI]) used for preparation of ion-gel film. (b) Schematic and (c) optical micrograph of the two-terminal device for characterization of electrical properties of hybrid dielectrics. (d) Current-voltage characteristics of hybrid dielectrics with various $\mathrm{Al}_{2} \mathrm{O}_{3}$ layer thicknesses. Inset: log-scale plot. (e) The variation of current density measured at $1 \mathrm{~V}$ as a function of $\mathrm{Al}_{2} \mathrm{O}_{3}$ layer thickness.

Meanwhile, in recent years, thin-film transistors (TFT) based on two-dimensional (2D) materials, such as hexagonal metal dichalcogenides, have been extensively studied for their potential applicability in emerging electronic and optoelectronic devices ${ }^{22-25}$. 2D material-based TFTs using ion gels as gate dielectrics have been reported to exhibit superior electrical properties, such as low threshold voltages, high carrier mobilities, transparency, and flexibility ${ }^{26-29}$. Braga et al. realized ambipolar transistors fabricated using exfoliated $\mathrm{WS}_{2}$ flakes with ionic liquid and proposed a method to determine the bandgap size directly ${ }^{30}$; Zhang et al. demonstrated the formation and detection of field-induced $p-n$ junctions in $\mathrm{MoS}_{2}$ EDLTs $^{31}$. Thus, ion gels as gate dielectrics for 2D material-based devices have several advantages, but their practicality must be improved to realize high-performance devices. Electric double-layer dielectrics, including ion gels, exhibit high leakage current levels between the gate and source/drain electrodes compared to those in inorganic-based gate dielectric ${ }^{32}$. For channel materials with greater gate leakage currents than intrinsic off-current levels leakage not only degrades the on/ off characteristics of the devices but also increases the threshold voltage. For example, the off-current level of previously reported monolayer $\mathrm{MoS}_{2}$ transistors was in the range of $10^{-10}-10^{-12} \mathrm{~A}^{33-36}$, which is less than the gate leakage current level of several nano-amperes at the operating voltage in ion-gel-gated devices ${ }^{32,37}$. Most reported ion-gel-gated transistors with $2 \mathrm{D}$ materials exhibit off-current levels exceeding the nano-ampere range, which might be greater than the intrinsic properties of the channel materials; in such cases, the transfer curves are expressed on linear rather than logarithmic scales. Therefore, it is important to reduce the leakage current through ion-gel dielectrics between the gate and source/drain electrodes to achieve higher-performance devices, particularly for channel materials with low off-current levels.

In this study, we propose a hybrid gate dielectric structure composed of an ion-gel film and ultra-thin $\mathrm{Al}_{2} \mathrm{O}_{3}$ passivation layer deposited by atomic layer deposition (ALD) to improve the electrical properties of the ion-gel-based 2D transistor, because $\mathrm{Al}_{2} \mathrm{O}_{3}$ films exhibit good leakage current properties and the ultra-thin $\mathrm{Al}_{2} \mathrm{O}_{3}$ layer can act as a leakage current barrier without causing significant capacitance drops $s^{38-40}$. Moreover, it is known that the ultra-thin $\mathrm{Al}_{2} \mathrm{O}_{3}$ films formed by ALD exhibit flexibility and transparency ${ }^{35,41}$, and therefore the hybrid dielectric structure could be suitable for application to flexible and transparent devices. To optimize the structure of the hybrid dielectrics, the variations in the electrical properties of the $\mathrm{Al}_{2} \mathrm{O}_{3}$ layer-modified ion gel film were systemically investigated as a function of the thickness of the $\mathrm{Al}_{2} \mathrm{O}_{3}$ passivation layer. Finally, the optimized dielectric structure was applied to a monolayer $\mathrm{MoS}_{2}$ transistor and compared to a transistor using a normal ion-gel gate dielectric to confirm the effect of the proposed hybrid dielectrics on the transistor performance.

\section{Results and Discussion}

The ion gel films were prepared from a solution of the triblock copolymer poly(styrene-block-methyl methacrylate-block-styrene) (PS-PMMA-PS) and the ionic liquid 1-ethyl-3-methylimi dazolium bis(trifluoromethylsulfonyl)imide ([EMIM][TFSI]), in ethyl propionate as an organic solvent. The PS-PMMA-PS and [EMIM][TFSI], with molecular structures as shown in Fig. 1a, are commonly used base materials to fabricate ion gels for EDLTs ${ }^{28,29}$. First, we fabricated simple two-terminal devices, as shown in Fig. 1b,c, to investigate the effect of $\mathrm{Al}_{2} \mathrm{O}_{3}$ passivation on the electrical properties of the ion-gel films. The ultra-thin $\mathrm{Al}_{2} \mathrm{O}_{3}$ layers were deposited by thermal ALD using $\mathrm{H}_{2} \mathrm{O}$ as an oxidant at the temperature of $350^{\circ} \mathrm{C}$. The reasons for determining the deposition conditions of $\mathrm{ALD}-\mathrm{Al}_{2} \mathrm{O}_{3}$ will be discussed later. Figure 1d shows the current $(I)$-voltage $(V)$ characteristics of the two-terminal devices according to the thicknesses of the inserted $\mathrm{Al}_{2} \mathrm{O}_{3}$ layers between the metal electrodes and ion gels. With the insertion of the $\mathrm{Al}_{2} \mathrm{O}_{3}$ passivation layer, the leakage current is remarkably decreased. When the dielectric film comprises only the ion gel without a passivation layer, the current level at $1 \mathrm{~V}$ is $\sim 10^{-9} \mathrm{~A}$, indicating difficulty in accurately measuring the on/off characteristics for EDLTs devices with off-current levels below the nano-ampere scale. As shown in Fig. 1e and the inset of Fig. 1d, the current levels through the hybrid dielectric films exponentially decrease as the $\mathrm{Al}_{2} \mathrm{O}_{3}$ thickness is increased to $10 \mathrm{~nm}$. This confirms that the leakage current can be effectively reduced by more than two and three orders of magnitude by inserting $\mathrm{Al}_{2} \mathrm{O}_{3}$ layers of only 3 and $5 \mathrm{~nm}$ in thickness, respectively. 
(a)

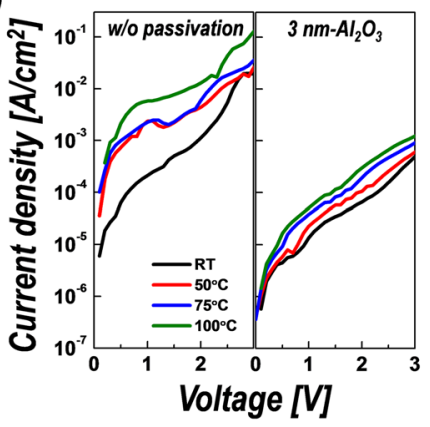

(b)

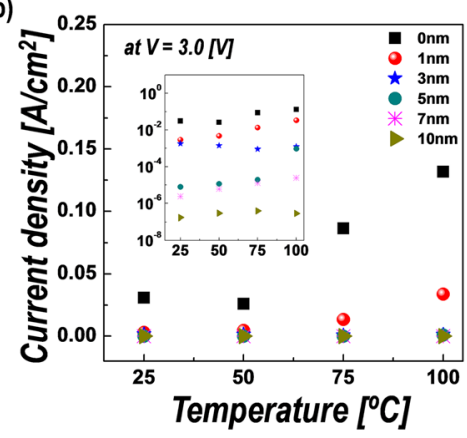

(c)
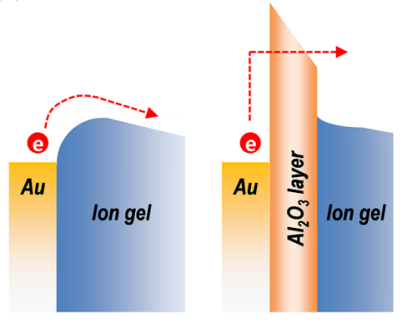

Figure 2. (a) $J-V$ characteristics of ion gel film without passivation and with 3-nm-thick $\mathrm{Al}_{2} \mathrm{O}_{3}$ passivation layer, measured from room temperature to $100^{\circ} \mathrm{C}$. (b) Temperature-dependent current density of hybrid structure with various thicknesses of $\mathrm{Al}_{2} \mathrm{O}_{3}$ passivation layer, measured at $3 \mathrm{~V}$ bias. Inset: log-scale plot. (c) Schematics of current mechanism at metal-ion gel and metal- $\mathrm{Al}_{2} \mathrm{O}_{3}$-ion gel interfaces.

Furthermore, we investigated the temperature dependence of the leakage current through the hybrid ion-gel films to study the change of current mechanism caused by the insertion of the $\mathrm{Al}_{2} \mathrm{O}_{3}$ layer. Figure $2 \mathrm{a}$ shows the current density $(J)$-voltage $(V)$ characteristics of the ion gel-based dielectric films without and with a 3-nm-thick $\mathrm{Al}_{2} \mathrm{O}_{3}$ passivation layer measured at the temperature range of room temperature to $100^{\circ} \mathrm{C}$. With the insertion of the ultra-thin $\mathrm{Al}_{2} \mathrm{O}_{3}$ layer, not only the overall leakage current level decreases, but also the temperature dependency of the current is decreased (data for samples with different passivation thicknesses is shown in Fig. S2 of the Supplementary Information). As indicated in Fig. 2b, the temperature dependency of the leakage current tends to decrease with increasing thickness of the $\mathrm{Al}_{2} \mathrm{O}_{3}$ passivation layer. Based on these observations, the current mechanism and the cause of the decrease in the leakage current by $\mathrm{Al}_{2} \mathrm{O}_{3}$ passivation can be explained as follows: In the absence of a passivation layer, the leakage current flows mainly by a thermionic mechanism, in which electrons flow over a specific barrier height between the metal electrode and the ion-gel dielectrics. However, the thin but large-bandgap $(\sim 8.9 \mathrm{eV}) \mathrm{Al}_{2} \mathrm{O}_{3}$ passivation layer located between the metal electrode and the ion gel acts as a leakage current barrier, preventing the flow of a direct thermionic current. Therefore, as the thickness of the passivation layer increases, the main current mechanism through the metal electrode to the ion gel-based dielectrics changes to electric field-induced tunneling, which is relatively insensitive to temperature, as indicated in the schematics in Fig. 2c. The results confirm that the leakage current characteristics are effectively improved by reducing the thermionic current in the dielectric structure with the placement of a large-bandgap $\mathrm{Al}_{2} \mathrm{O}_{3}$ passivation layer.

Meanwhile, although the leakage current characteristics of the device using a hybrid dielectric structure of ion gel with an $\mathrm{Al}_{2} \mathrm{O}_{3}$ passivation layer can be improved, the dielectric constant of $\mathrm{Al}_{2} \mathrm{O}_{3}$ is relatively low $(\sim 9)$, which can degrade the dielectric properties of the device. Therefore, we investigated the dielectric properties of ion gel-based hybrid dielectrics as a function of the thickness of the inserted $\mathrm{Al}_{2} \mathrm{O}_{3}$ layer. The capacitance density of the ion gel without passivation layer was $\sim 10.8 \mu \mathrm{F} / \mathrm{cm}^{-2}$, which is comparable to those of previously reported gels $\left(\sim 5-10 \mu \mathrm{F} / \mathrm{cm}^{-2}\right)^{4,29,37}$. The capacitance slightly increased by the insertion of an $\mathrm{Al}_{2} \mathrm{O}_{3}$ layer with the thickness of $\sim 3 \mathrm{~nm}$ and then continuously decreased with further increases in the inserted $\mathrm{Al}_{2} \mathrm{O}_{3}$ thickness. The decrease in capacitance for relatively thick $(>5 \mathrm{~nm}) \mathrm{Al}_{2} \mathrm{O}_{3}$ layers can be explained by the relatively small dielectric constant of $\mathrm{Al}_{2} \mathrm{O}_{3}$ films, but it is difficult to understand the increase in capacitance for 3-nm-thick $\mathrm{Al}_{2} \mathrm{O}_{3}$-inserted ion-gel films. However, it was previously reported that self-discharge at the charged state in electric double-layer capacitors, caused by high leakage currents, could cause capacitance drops ${ }^{42,43}$. Therefore, the increase in capacitance for the sample with the 3 -nm-thick $\mathrm{Al}_{2} \mathrm{O}_{3}$ layer was attributed to the decrease in leakage current by the insertion of the leakage current barrier. It is also observed that the frequency dependence of the capacitance tends to decrease with increases in the thickness of the $\mathrm{Al}_{2} \mathrm{O}_{3}$ passivation layer (see inset of Fig. 3). The most important point is that, although the low-dielectric-constant layer is inserted, the capacitance is not degraded until the $\mathrm{Al}_{2} \mathrm{O}_{3}$ thickness is increased to $\sim 5 \mathrm{~nm}$. Therefore, it can be expected that the hybrid gate structure of ion-gel dielectrics with ultra-thin $\mathrm{Al}_{2} \mathrm{O}_{3}$ passivation layers could effectively improve the transistor characteristics of EDLTs by reducing the gate leakage current without inducing capacitance drops.

Finally, the hybrid gate dielectric of ion-gel film with an ultra-thin $\mathrm{Al}_{2} \mathrm{O}_{3}$ passivation layer was applied to $\mathrm{MoS}_{2}$ EDLTs to confirm the effect of the proposed gate structure on the performance of the $\mathrm{MoS}_{2}$ transistors. Figure $4 \mathrm{a}, \mathrm{b}$ show the schematic and optical micrograph of the fabricated $\mathrm{MoS}_{2}$ EDLT structure using the hybrid gate dielectric, respectively. Monolayer $\mathrm{MoS}_{2}$ crystals directly grown on a $\mathrm{SiO}_{2} / \mathrm{Si}$ substrate by chemical vapor deposition (CVD) were used as a transistor channel; source/drain and side gate electrodes were defined by the lift-off method. After formation of electrodes, 3-nm-thick $\mathrm{Al}_{2} \mathrm{O}_{3}$ layers were deposited by thermal ALD at $350{ }^{\circ} \mathrm{C}$. ALD $\mathrm{Al}_{2} \mathrm{O}_{3}$ films are usually obtained using $\mathrm{H}_{2} \mathrm{O}, \mathrm{O}_{3}$, or $\mathrm{O}_{2}$ plasma as the oxidant at the temperature range of 50 to $400{ }^{\circ} \mathrm{C}$. In our case, we used $\mathrm{H}_{2} \mathrm{O}$ vapor as an oxidant to prevent damage to the $\mathrm{MoS}_{2}$ channel by ozone or $\mathrm{O}_{2}$ plasma. In addition, because ideal van der Waals 2D materials do not have dangling surface bonds, the precursor molecules for ALD growth were infrequently adsorbed on the 2D materials, so that thin film growth by ALD could be suppressed on the $2 \mathrm{D}$ materials. Therefore, the $\mathrm{ALD}-\mathrm{Al}_{2} \mathrm{O}_{3}$ layer could be selectively deposited except on the $2 \mathrm{D}$ material surface without additional etching processes. However, the CVD-grown $\mathrm{MoS}_{2}$ could contain defect sites where the precursor can chemosorb; therefore, the ALD process for depositing the $\mathrm{Al}_{2} \mathrm{O}_{3}$ passivation layer was conducted at the temperature of $350{ }^{\circ} \mathrm{C}$ to minimize the chemisorption of precursors on the $\mathrm{MoS}_{2}$ 


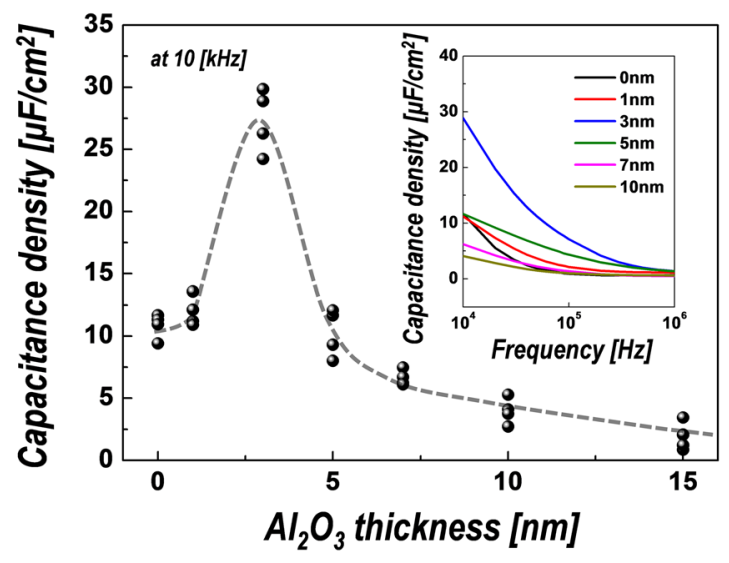

Figure 3. The variation of the capacitance density of two-terminal device with hybrid dielectrics depending on $\mathrm{Al}_{2} \mathrm{O}_{3}$ layer thickness with frequency of $10 \mathrm{kHz}$. Inset: frequency dependence of the capacitance for different $\mathrm{Al}_{2} \mathrm{O}_{3}$ thickness.

(a)

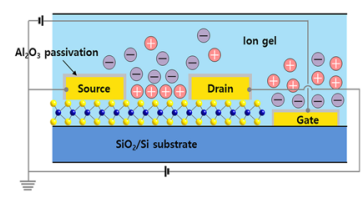

(b)

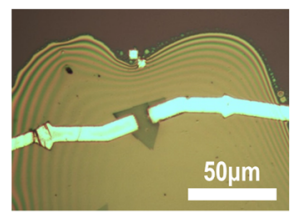

(c)

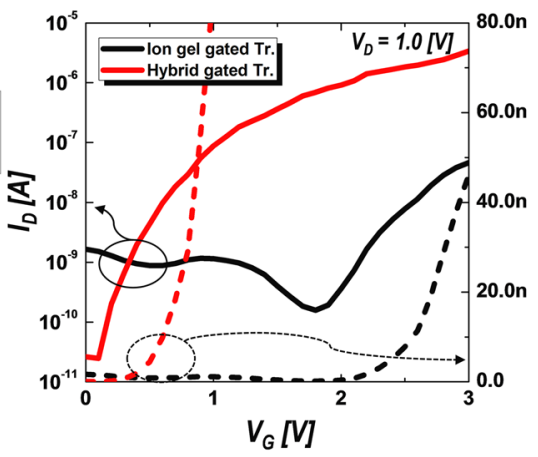

(d)

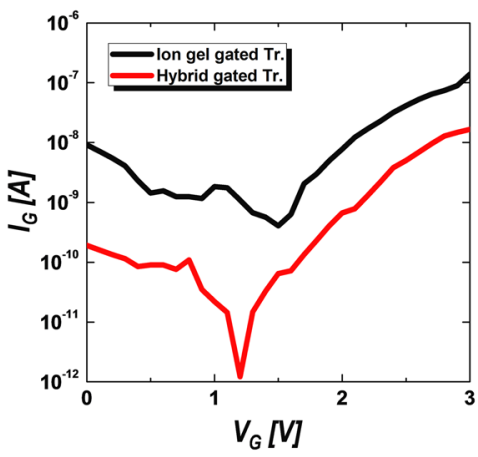

Figure 4. (a) Schematic and (b) optical micrograph of the hybrid-structure EDLTs fabricated using monolayer single-crystalline $\mathrm{MoS}_{2}$. (c) Transfer curve and (d) gate leakage current (absolute values) of the $\mathrm{MoS}_{2}$ EDLTs without passivation (ion gel gated Tr.) and with 3-nm-thick $\mathrm{Al}_{2} \mathrm{O}_{3}$ passivation layer (Hybrid gated Tr.) measured at drain voltage of $1.0 \mathrm{~V}$ under room temperature. Solid line and dashed line in transfer graph indicate the logarithmic-scale (left-side axis) and linear-scale (right-side axis) value of drain current, respectively.

surface ${ }^{44}$. After the deposition of the 3-nm-thick $\mathrm{Al}_{2} \mathrm{O}_{3}$ layer by $\mathrm{ALD}$ on the $\mathrm{MoS}_{2}$ crystals, it was confirmed that the $\mathrm{Al}_{2} \mathrm{O}_{3}$ layer was rarely deposited on the surface of the $\mathrm{MoS}_{2}$ crystals by atomic force microscopy analysis (see Fig. S3 of the Supplementary Information). In fact, since no degradation of capacitance was observed for inserted $\mathrm{Al}_{2} \mathrm{O}_{3}$ layer thicknesses below $\sim 5 \mathrm{~nm}$, it can be expected that even if the ultra-thin $\mathrm{Al}_{2} \mathrm{O}_{3}$ is partially deposited on $\mathrm{MoS}_{2}$, the transistor performance would not be negatively affected. The room-temperature transfer characteristics $\left(I_{D}-V_{G}\right)$ of the $\mathrm{MoS}_{2}$ EDLTs measured at the source-drain voltage $\left(V_{D}\right)$ of $1.0 \mathrm{~V}$ with and without $\mathrm{Al}_{2} \mathrm{O}_{3}$ passivation are indicated in Fig. 4c. For comparison, regardless of with and without passivation, the channel length and width were fixed at 10 and $10 \mu \mathrm{m}$, respectively. Whereas the on/off ratio of the ion gel-only gated transistor is $\sim 10^{2}$ because of the relatively high off-current level of $\sim 10^{-9} \mathrm{~A}$, the on/off ratio of the hybrid-gated transistor is remarkably increased to $\sim 10^{5}$. Moreover, it is observed that the threshold voltage is decreased by more than $2 \mathrm{~V}$, and the subthreshold swing (SS) is also improved by inserting the ultra-thin $\mathrm{Al}_{2} \mathrm{O}_{3}$ layer between the electrodes. These improvements in the electrical properties of the transistor by the $\mathrm{Al}_{2} \mathrm{O}_{3}$ passivation layer are mainly attributed to the reduction of the leakage current between the gate and source/drain electrodes. By applying the hybrid gate structure of ion-gel dielectrics with 3 -nm-thick $\mathrm{Al}_{2} \mathrm{O}_{3}$ passivation layers, as shown in Fig. 4d, the gate leakage current is significantly decreased by about two orders of magnitude (from several nano-amperes to several tens of pico-amperes at $I_{D}=1.0 \mathrm{~V}$ ), which well corresponds with the off-current level of each transistor. In addition, the on-current level of the hybrid gated EDLTs was also greatly increased by $\mathrm{Al}_{2} \mathrm{O}_{3}$ passivation, which might be attributed to the reduced dielectric loss from the reduced gate leakage current.

\section{Conclusions}

In this study, we systematically investigated the effect of inserting an $\mathrm{Al}_{2} \mathrm{O}_{3}$ passivation layer between the ion-gel dielectric film and metal electrode on the electrical properties of a transistor to propose a hybrid ion gel-based gate structure with improved properties. As the thickness of the inserted $\mathrm{Al}_{2} \mathrm{O}_{3}$ layer was increased, the leakage current through the ion gel-based dielectrics was exponentially decreased (more than two orders of magnitude even with an ultra-thin $\mathrm{Al}_{2} \mathrm{O}_{3}$ layer of $3 \mathrm{~nm}$ in thickness). Moreover, no degradation of capacitance was observed for 
inserted $\mathrm{Al}_{2} \mathrm{O}_{3}$ layer thicknesses below $\sim 5 \mathrm{~nm}$. The hybrid structured gate dielectric based on the ion-gel film with a 3-nm-thick $\mathrm{Al}_{2} \mathrm{O}_{3}$ layer was applied to a monolayer $\mathrm{MoS}_{2}$ transistor. The performance of the transistor was compared to that of another transistor using a normal ion-gel gate dielectric. Most transistor properties, such as the on/ off ratio, threshold voltage, and subthreshold swing, were improved by the remarkable decrease of the gate leakage current. The proposed hybrid-structured gate dielectrics could be applied to versatile devices, especially with transparency and flexibility, based on EDLTs using various channel materials, as well as 2D material-based transistors.

\section{Methods}

Preparation of ion gel. The ion gel was prepared from a solution of a triblock copolymer, poly(styrene-block-methyl methacrylate-block-styrene) (PS-PMMA-PS, Polymer Source Inc.), and an ionic liquid, 1-ethyl-3-methylimi dazolium bis(trifluoromethylsulfonyl)imide ([EMIM][TFSI], Merck), in ethyl propionate as the organic solvent ${ }^{28,29}$. The polymer, ionic liquid, and solvent were mixed with the weight ratio of 0.5:9.5:20, and then the mixture was sonicated to obtain a homogeneous solution. This solution was drop-casted onto the metal electrodes of the device. Through annealing at $120^{\circ} \mathrm{C}$ for $10 \mathrm{~min}$, the solvent was evaporated and an ion gel film was formed through the physical association of the polymer blocks in the ionic liquid.

Deposition of ultra-thin $\mathrm{Al}_{2} \mathrm{O}_{3}$ layer. To enhance the electrical properties of the gate dielectric based on the ion gel, an ultra-thin $\mathrm{Al}_{2} \mathrm{O}_{3}$ layer, deposited by $\mathrm{ALD}$ at $350{ }^{\circ} \mathrm{C}$, was introduced between the metal electrode and ion-gel interface. Trimethylaluminum (TMA, $\left.\mathrm{Al}\left(\mathrm{CH}_{3}\right)_{3}\right)$ and water vapor $\left(\mathrm{H}_{2} \mathrm{O}\right)$ were used as precursor and oxidant, respectively; both canisters containing TMA and $\mathrm{H}_{2} \mathrm{O}$ were maintained at the temperature of $10^{\circ} \mathrm{C}$. The deposition process was performed under saturation conditions. One deposition cycle consisted of the exposure of TMA for $0.5 \mathrm{~s}$, purging with $\mathrm{N}_{2}$ for $5 \mathrm{~s}$, exposure of $\mathrm{H}_{2} \mathrm{O}$ for $0.5 \mathrm{~s}$, and purging with $\mathrm{N}_{2}$ for $5 \mathrm{~s}$.

Device fabrication and characterization. To investigate the effect of $\mathrm{Al}_{2} \mathrm{O}_{3}$ passivation on the electrical properties, a simple two-terminal device was fabricated on $\mathrm{SiO}_{2} / \mathrm{Si}$ substrate. First, two electrodes were formed from 10-nm Ti/50-nm Au layers via a lift-off method using photolithography (Photo resist: AZ5214, AZ Electronic Materials) and e-beam evaporation. The hybrid gate dielectric was formed via the deposition of ultra-thin $\mathrm{Al}_{2} \mathrm{O}_{3}$ layers with thicknesses of $0-10 \mathrm{~nm}$, followed by the drop-casting of the ion gel. Meanwhile, to apply the hybrid gate dielectric to field-effect transistor (FET) devices, $\mathrm{MoS}_{2}$ transistors were fabricated using monolayer crystals synthesized by CVD (see Fig. S1 of the Supplementary Information for more details on the synthesis process). Similar to the above method, source/drain and side gate electrodes were formed from 10-nm Ti/50-nm Au layers by a lift-off method. In this case, however, electron-beam lithography using poly(methyl methacrylate) (PMMA) was used instead of photolithography, because the source/drain electrodes required greater accuracy in formation on the $\mathrm{MoS}_{2}$ crystal. Finally, for comparison, two types of ion gel-based gate dielectrics were formed, one without passivation and the other with a 3-nm-thick $\mathrm{Al}_{2} \mathrm{O}_{3}$ passivation layer. After the device fabrication, capacitance-voltage $(C-V)$, current-voltage $(I-V)$, and FET transfer characteristics measurements were performed in a dark probe station using a semiconductor characterization system (Keithley, 4200-SCS).

\section{References}

1. Yuan, H. et al. Electrostatic and Electochemical Nature of Liquid-Gated Electric-Double-Layer Transistor Based on Oxide Semiconductor. J. Am. Chem. Soc. 132, 18402-18407 (2010).

2. Du., H., Lin, X., Xu, Z. \& Chu, D. Electric Double-Layer Transistors: A Review of Recent Progress. J. Mater. Sci. 50, 5641-5673 (2015).

3. Perera, M. M. et al. Improved Carrier Mobility in Few-Layer $\mathrm{MoS}_{2}$ Field-Effect Transistors with Ionic-Liquid Gating. ACS Nano 7, 4449-4458 (2013).

4. Ye, J. T. et al. Liquid-Gated Interface Superconductivity on an Atomically Flat Film. Nat. Mater. 9, 125-128 (2010).

5. Yuan, H. et al. High-Density Carrier Accumulation in ZnO Field-Effect Transistor Gated by Electric Double Layer of Ionic Liquid. Adv. Funct. Mater. 19, 1046-1053 (2009)

6. Fujimoto, T. \& Awaga, K. Electric-Double-Layer Field-Effect Transistor with Ionic Liquids. Phys. Chem. Chem. Phys. 15, 8983-9006 (2013).

7. Zhang, Y., Ye, J., Matsuhashi, Y. \& Iwasa, Y. Ambipolar MoS 2 Thin Flake Transistor. Nano Lett. 12, 1136-1140 (2012).

8. Allain, A. \& Kis, A. Electron and Hole Mobility in Single-Layer WSe. ACS Nano 8, 7180-7185 (2014).

9. Xia, Y., Cho, J. H., Lee, J., Ruden, P. P. \& Frisbie, C. D. Comparison of the Mobility-Carrier Density Relation in Polymer and SingleCrystal Organic Transistors Employing Vacuum and Liquid Gate Dielectrics. Adv. Mater. 21, 2174-2179 (2009).

10. Yanagi, K. et al. Tuning of the Thermoelectric Properties of One-Dimensional Material Networks by Electron Double Layer Techniques Using Ionic Liquids. Nano Lett. 14, 6437-6442 (2014).

11. Choi, J.-H., Xie, W., Gu, Y., Frisbie, C. D. \& Lodge, T. P. Single Ion Conducting, Polymerized Ionic Liquid Triblock Copolymer Films: High Capacitance Electrolyte Gates for n-type Transistors. ACS Appl. Mater. Interface 7, 7294-7302 (2015).

12. Yuan, H. T. et al. Liquid-Gated Electric-Double-Layer Transistor on Layered Metal Dichalcogenide, SnS 2 . Appl. Phys. Lett. 98 , $012102(2011)$.

13. Lei, B. et al. Evolution of High-Temperature Superconductivity from a Low- $\mathrm{T}_{\mathrm{c}}$ Phase Tuned by Carrier Concentration in FeSe Thin Flakes. Phys. Rev. Lett. 116, 077002 (2016).

14. Lee, J., Panzer, M. J., He, Y., Lodge, T. P. \& Frisbie, C. D. Ion Gel Gated Polymer Thin-Film Transistors. J. Am. Chem. Soc. 129, 4532-4533 (2007).

15. Lee, J. et al. Ion Gel-Gated Polymer Thin-Film Transistors: Operating Mechanism and Characterization of Gate Dielectric Capacitance, Switching Speed, and Stability. J. Phys. Chem. C. 113, 8972-8981 (2009).

16. Xu, F. et al. Highly Stretchable Carbon Nanotube Transistors with Ion Gel Gate Dielectrics. Nano Lett. 14, 682-686 (2014).

17. Oh, G. et al. Graphene/Pentacene Barristor with Ion-Gel Gate Dielectric: Flexible Ambipolar Transistor with High Mobility and On/ Off Ratio. ACS Nano 9, 7515-7522 (2016).

18. Moon, H. C., Kim, C.-H., Lodge, T. P. \& Frisbie, C. D. Multicolored, Low-Power, Flexible Electrochromic Devices Based on Ion Gels. ACS Appl. Mater. Interface 8, 6252-6260 (2016).

19. Wu, M.-Y. et al. Highly stretchable carbon nanotube transistors enabled by buckled ion gel gate dielectrics. Appl. Phys. Lett. 107, 053301 (2015). 
20. Cho, J. H. et al. Printable Ion-Gel Gate Dielectrics for Low-Voltage Polymer Thin-Film Transistors on Plastic. Nat. Mater. 7, 900-906 (2008).

21. Lee, S.-K. et al. Photopatternable Ion Gel Gated Graphene Transistors and Inverters on Plastic. Nanotechnology 25, 014002 (2014).

22. Radisavljevic, B., Radenovic, A., Brivio, J., Giacometti, V. \& Kis, A. Single-Layer $\mathrm{MoS}_{2}$ Transistor. Nat. Nanotechnol. 6, 147-150 (2011).

23. Wang, Q. H., Kalantar-Zadeh, K., Kis, A., Coleman, J. N. \& Strano, M. S. Electronics and Optoelectronics of Two-Dimensional Transition Metal Dichalcogenides. Nat. Nanotechnol. 7, 699-712 (2012).

24. Jariwala, D., Sangwan, V. K., Lauhon, L. J., Marks, T. J. \& Hersam, M. C. Emerging Device Applications for Semiconducting TwoDimensional Transition Metal Dichalcogenides. ACS Nano. 8, 1102-1120 (2014).

25. Akinwande, D., Petrone, N. \& Hone, J. Two-Dimensional Flexible Nanoelectronics. Nat. Commun. 5, 5678 (2014).

26. Pu, J. et al. Highly Flexible and High-Performance Complementary Inverters of Large-Area Transition Metal Dichalcogenide Monolayers. Adv. Mater. 28, 4111-4119 (2016).

27. Choi, Y. et al. Low-Voltage Complementary Electronics from Ion-Gel-Gated Vertical Van der Waals Heterostructures. Adv. Mater. 28, 3742-3748 (2016).

28. $\mathrm{Pu}$, J. et al. Highly Flexible $\mathrm{MoS}_{2}$ Thin-Film Transistors with Ion Gel Dielectrics. Nano Lett. 12, 4013-4017 (2012).

29. Pu, J., Zhang, Y., Wada, Y., Wang, J. T.-W. \& Li, L. J. Fabrication of Stretchable $\mathrm{MoS}_{2}$ Thin-Film Transistor Using Elastic Ion Gel Gate Dielectrics. Appl. Phys. Lett. 103, 023505 (2013).

30. Braga, D., Lezama, I. G., Berger, H. \& Morpurgo, A. F. Quantitative Determination of the Band Gap of $\mathrm{WS}_{2}$ with Ambipolar Ionic Liquid-Gated Transistors. Nano Lett. 12, 5218-5223 (2012).

31. Zhang, Y. J., Ye, J. T., Yomogida, Y., Takenobu, T. \& Iwasa, Y. Formation of a Stable $p-n$ Junction in a Liquid-Gated $M_{2}$ Ambipolar $_{2}$ Transistor. Nano Lett. 13, 3023-3028 (2013).

32. Fabiano, S., Crispin, X. \& Berggren, M. Ferroelectric Polarization Induces Electric Double Layer Bistability in Electrolyte-Gated Field-Effect Transistors. ACS Appl. Mater. Interface 6, 438-442 (2014).

33. Lopez-Sanchez, O., Lembke, D., Kayci, M., Radenovic, A. \& Kis, A. Ultrasensitive Photodetectors Based on Monolayer MoS 2. Nat. Nanotechnol. 8, 497-501 (2013).

34. Lee, Y.-H. et al. Synthesis of Large-Area MoS 2 Atomic Layers with Chemical Vapor Deposition. Adv. Mater. 24, 2320-2325 (2012).

35. Chang, H.-Y. et al. High-Performance, Highly Bendable $\mathrm{MoS}_{2}$ Transistors with High-K Dielectrics for Flexible Low-Power Systems. ACS Nano. 7, 5446-5452 (2013).

36. Park, W. et al. Photoelectron Spectroscopic Imaging and Device Applications of Large-Area Patternable Single-Layer $\mathrm{MoS}_{2}$ Synthesized by Chemical Vapor Deposition. ACS Nano. 8, 4961-4968 (2014).

37. Cho, J. H. et al. High-Capacitance Ion Gel Gate Dielectrics with Faster Polarization Response Times for Organic Thin Film Transistor. Adv. Mater. 20, 686-690 (2008).

38. Ahn, J.-H., Kim, J.-Y., Jeong, S.-J. \& Kwon, S.-H. Effect of $\mathrm{Al}_{2} \mathrm{O}_{3}$ Insertion on the Electrical Properties of $\mathrm{SrTiO}_{3}$ Thin Films: A Comparison between $\mathrm{Al}_{2} \mathrm{O}_{3}$-doped $\mathrm{SrTiO}_{3}$ and $\mathrm{SrTiO}_{3} / \mathrm{Al}_{2} \mathrm{O}_{3} / \mathrm{SrTiO}_{3}$. Mater. Res. Bull. 64, 1-5 (2015).

39. Martin, D. et al. Mesoscopic Analysis of Leakage Current Suppression in $\mathrm{ZrO}_{2} / \mathrm{Al}_{2} \mathrm{O}_{3} / \mathrm{ZrO}_{2}$ Nano-Laminates. J. Appl. Phys. 113, $194103(2013)$.

40. Wu, Y.-H. et al. High density metal-insulator-metal capacitor based on $\mathrm{ZrO}_{2} / \mathrm{Al}_{2} \mathrm{O}_{3} / \mathrm{ZrO}_{2}$ laminate dielectric. Appl. Phys. Lett. 93, 033511 (2008).

41. Xiao, W. et al. A flexible transparent gas barrier film employing the method of mixing $\mathrm{ALD} / \mathrm{MLD}$-grown $\mathrm{Al}_{2} \mathrm{O}_{3}$ and alucone layers. Nanoscale Res. Lett. 10, 130 (2015).

42. Ishikawa, M., Morita, M., Ihara, M. \& Matsuda, Y. Electric Double-layer Capacitor Composed of Activated Carbon Fiber Cloth Electrodes and Solid Polymer Electrolytes Containing Alkylammonium Salts. J. Electrochem. Soc. 141, 1730-1734 (1994).

43. Jang, J. H., Yoon, S., Ka, B. H., Jung, Y.-H. \& Oh, S. M. Complex Capacitance Analysis on Leakage Current Appearing in Electric Double-layer Capacitor Carbon Electrode. J. Electrochem. Soc. 151, A1418-A1422 (2005).

44. Park, T. et al. Atomic Layer Deposition of $\mathrm{Al}_{2} \mathrm{O}_{3}$ on $\mathrm{MoS}_{2}, \mathrm{WS}_{2}, \mathrm{WSe}_{2}$, and h-BN: Surface Coverage and AdsorptionEnergy. RCS Adv. 7, 884-889 (2017).

\section{Acknowledgements}

This work was supported by National Research Foundation of Korea (NRF) grant funded by the Korea government (MSIP) (No. 2016R1C1B2007336 and No. 2017R1A2B4004560). This work was also supported by New Technology Commercialization Support Program for Young Researchers (2016) funded by the Busan metropolitan city and Busan Institute of S\&T Evaluation and Planning (BISTEP).

\section{Author Contributions}

S.-H.K. and J.-H.A. conceived the idea, designed the experiments. C.-M.H. prepared the CVD-grown $\mathrm{MoS}_{2}$

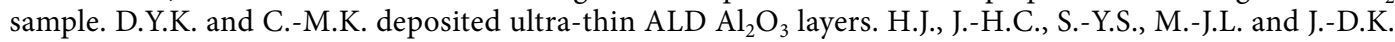
fabricated the ion gel devices and performed the electrical measurement. H.J., J.-H.C. and H.-S.M. analyzed the data under the supervision of J.-H.A. The manuscript was written by H.J., J.-H.C., C.-M. H. and J.-H.A. All the authors reviewed and commented on the manuscript.

\section{Additional Information}

Supplementary information accompanies this paper at https://doi.org/10.1038/s41598-017-14649-6.

Competing Interests: The authors declare that they have no competing interests.

Publisher's note: Springer Nature remains neutral with regard to jurisdictional claims in published maps and institutional affiliations.

Open Access This article is licensed under a Creative Commons Attribution 4.0 International License, which permits use, sharing, adaptation, distribution and reproduction in any medium or format, as long as you give appropriate credit to the original author(s) and the source, provide a link to the Creative Commons license, and indicate if changes were made. The images or other third party material in this article are included in the article's Creative Commons license, unless indicated otherwise in a credit line to the material. If material is not included in the article's Creative Commons license and your intended use is not permitted by statutory regulation or exceeds the permitted use, you will need to obtain permission directly from the copyright holder. To view a copy of this license, visit http://creativecommons.org/licenses/by/4.0/.

(C) The Author(s) 2017 